\title{
CResearch Square

\section{An Investigation Into The Mathematical and Physical Origins of The Fine-Structure Constant}

\section{Lamont Williams ( $\nabla$ williams_lamont@msn.com )}

National Institutes of Health

\section{Research Article}

Keywords: Fine-structure constant, electromagnetic coupling constant, universal physical constants, dimensionless physical constants, mathematical constants, electron spin g-factor, electron, muon, tau anomalous magnetic moment

Posted Date: November 16th, 2021

DOI: https://doi.org/10.21203/rs.3.rs-1059795/v1

License: (1) This work is licensed under a Creative Commons Attribution 4.0 International License. Read Full License 
1 An Investigation into the Mathematical and Physical Origins of the Fine-Structure Constant

2

3

4

5

6

7

8

9

10

11

12

13

14

15

16

17

18

19

20

21

22

23

25

26

27

28

30

31

32

33
Lamont Williams

National Institutes of Health

Center for Scientific Review

Bethesda, MD 20817

609-280-2440 (cell)

lamont.williams@nih.gov

williams_lamont@msn.com

ORCID:

https://orcid.org/0000-0003-0768-8437

Date: November 8, 2021
24

29

9

0

1

2

23

5

6

7

28

2 
34 An Investigation into the Mathematical and Physical Origins of the Fine-Structure Constant

\section{Abstract}

38 The fine-structure constant, $\alpha$, unites fundamental aspects of electromagnetism,

39 quantum physics, and relativity. As such, it is one of the most important constants in

40 nature. However, why it has the value of approximately $1 / 137$ has been a mystery since

41 it was first identified more than 100 years ago. To date, it is an ad hoc feature of the

42 Standard Model, as it does not appear to be derivable within that body of work - being

43 determined solely by experimentation. This report presents a mathematical formula for

$44 \alpha$ that results in an exact match with the currently accepted value of the constant. The

45 formula requires that a simple corrective term be applied to the value of one of the

46 factors in the suggested equation. Notably, this corrective term, at approximately 0.023 ,

47 is similar in value to the electron anomalous magnetic moment value, at approximately

480.0023 , which is the corrective term that needs to be applied to the g-factor in the

49 equation for the electron spin magnetic moment. In addition, it is shown that the

50 corrective term for the proposed equation for $\alpha$ can be derived from the anomalous

51 magnetic moment values of the electron, muon, and tau particle - values that have

52 been well established through theory and/or experimentation. This supports the notion

53 that the corrective term for the $\alpha$ formula is also a real and natural quantity. The

54 quantum mechanical origins of the lepton anomalous magnetic moment values suggest

55 that there might be a quantum mechanical origin to the corrective term for $\alpha$ as well.

56 This possibility, as well as a broader physical interpretation of the value of $\alpha$, is explored. 
57 Keywords: Fine-structure constant; electromagnetic coupling constant; universal

58 physical constants; dimensionless physical constants; mathematical constants; electron

59 spin g-factor; electron, muon, tau anomalous magnetic moment

60

\section{1. Introduction}

62 The fine-structure constant, $\alpha$, also called Sommerfeld's constant, and the

63 electromagnetic coupling constant, represents the strength of the interaction between

64 electrically charged elementary particles. At times referred to as a "magic number" and

65 "the most important number in physics," it unites fundamental aspects of

66 electromagnetism (elementary electric charge, $e$ ), quantum physics (reduced Planck's

67 constant, $\hbar$ ), and relativity (speed of light, $c$ ), as well as the electric permittivity of free

68 space $\left(\varepsilon_{0}\right)$ :

$$
\alpha=\frac{e^{2}}{4 \pi \varepsilon_{0} \hbar c} .
$$

70 Unfortunately, why $\alpha$ has the value it has, at approximately $1 / 137$, has been a mystery,

71 since it was identified by Arnold Sommerfeld in 1916. A fundamental mathematical

72 formula for the constant leading to a match with experimental results has been elusive,

73 particularly as $\alpha$ has appeared to have no connection to mathematical constants. In the

74 Standard Model, the number currently stands as an ad hoc value that must be

75 determined experimentally.

76 This study presents a mathematical formula for $\alpha$ that does involve mathematical

77 constants, and that leads to an exact match with the current recommended value of $\alpha$, 
78 as determined by the Committee on Data for Science and Technology (CODATA, 2018)

79 [1]. It is also shown how the formula is related to the well-established formula of the

80 electron spin magnetic moment, particularly the electron spin g-factor $\left(g_{e}\right)$ component

81 of the equation. It is further shown how the formula is mathematically linked to the

82 anomalous magnetic moment values of the charged leptons - values that have been

83 established through theory and/or experimentation. Additionally, a physical

84 interpretation of the suggested formula for $\alpha$ is presented, including the possibility of

85 there being a quantum mechanical contribution to the constant's value.

\section{2. Analysis and Discussion}

87 The equation for the electron's spin magnetic moment provides important insight into

88 the possible mathematical underpinnings of $\alpha$. The z-component of the magnetic

89 moment can be calculated as follows:

$$
\mu_{z}= \pm 1 / 2\left|g_{e}\right| \mu_{B}
$$

92 where $g_{e}$ is the electron spin g-factor, a dimensionless value, and $\mu_{B}$ is the Bohr

93 magneton, a unit of magnetic moment.

94 Paul Dirac predicted $g_{e}$ to have a value of -2 [2]. However, experimentation has shown

95 the value to actually be closer to $-2.00231930436256(35)$. The correction needed on the 96 value of 2 can be symbolized as follows:

97 
101 where $\alpha_{e}$ is $0.00115965218128(18)$ and is referred to as the electron's anomalous

102 magnetic moment. The anomaly arises from quantum effects at the particle level that

103 cause the value of $g_{e}$ to slightly exceed 2 . The full value of $g_{e}$ can be formulated well

104 through perturbative quantum field theory techniques, thus far matching up to 10

105 significant digits of the experimentally determined value $[3,4,5,6]$.

106 The principal idea here is that one of the factors in the equation for $\mu_{z}$ (specifically $g_{e}$ )

107 requires the addition of a small corrective, or anomalous, term -2 times

$1080.00115965218128(18)$, or $0.00231930436256(35)-$ to obtain the true value of $g_{e}$ and

109 thereby $\mu_{\mathrm{z}}$.

110 A similar situation appears to arise in the setting of $\alpha$. That is, as there is an anomalous

111 value associated with the electron's magnetic field that must be accounted for to

112 calculate the accurate value of the spin, there also appears to be an anomalous value

113 associated with the electron's electric field that must be accounted for in the calculation

114 of $\alpha$. The concept of electric field lines can help in initial steps to identify the anomalous

115 electric field value.

116 The electric force between two electrons - or, for ease, an electron and positron,

117 before annihilation - can be depicted via classical field lines, Fig. 1. 


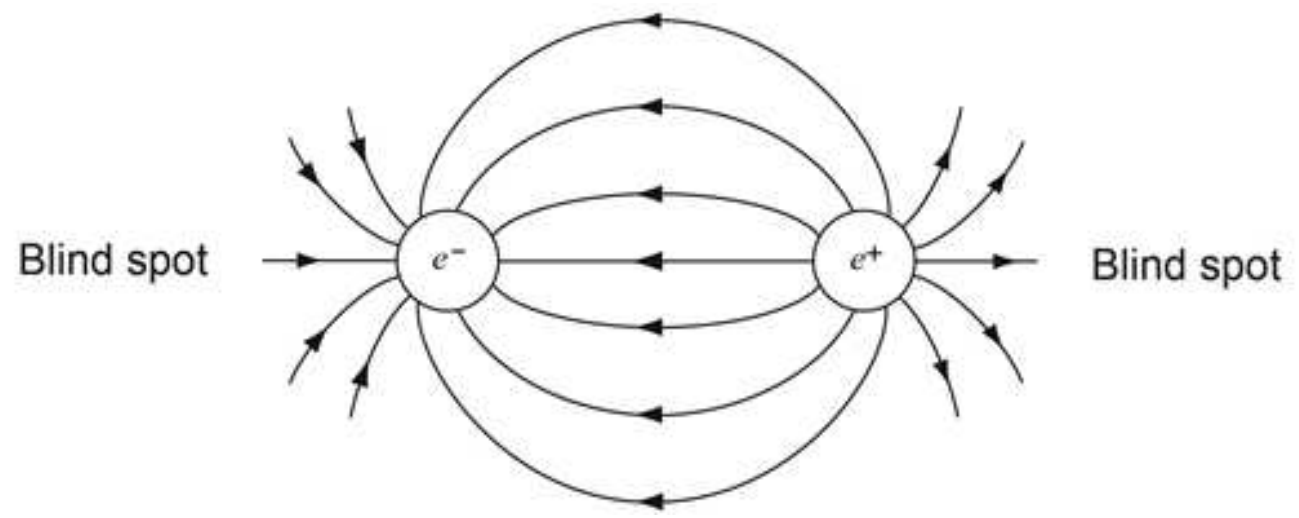

119 Fig. 1|The electric interaction between an electron and positron (before annihilation),

120 as depicted using classical field lines. On the far side of the particles, there are field

121 lines that do not take part in the interaction, with the field lines simply extending into

122 space, representing a blind spot in the field.

123 The field lines represent the interaction, and thus the coupling of the particles - i.e.,

124 how strong that force is. An increase in the density of the field lines would represent an

125 increase in the coupling or strength of the force. However, as shown in Fig. 1, on the far

126 side of the particles, there are field lines that do not take part in the interaction, with

127 the field lines simply extending into space. This can be viewed on a macroscopic level

128 using the magnetic field analogue of the electric field (Fig. 2). 


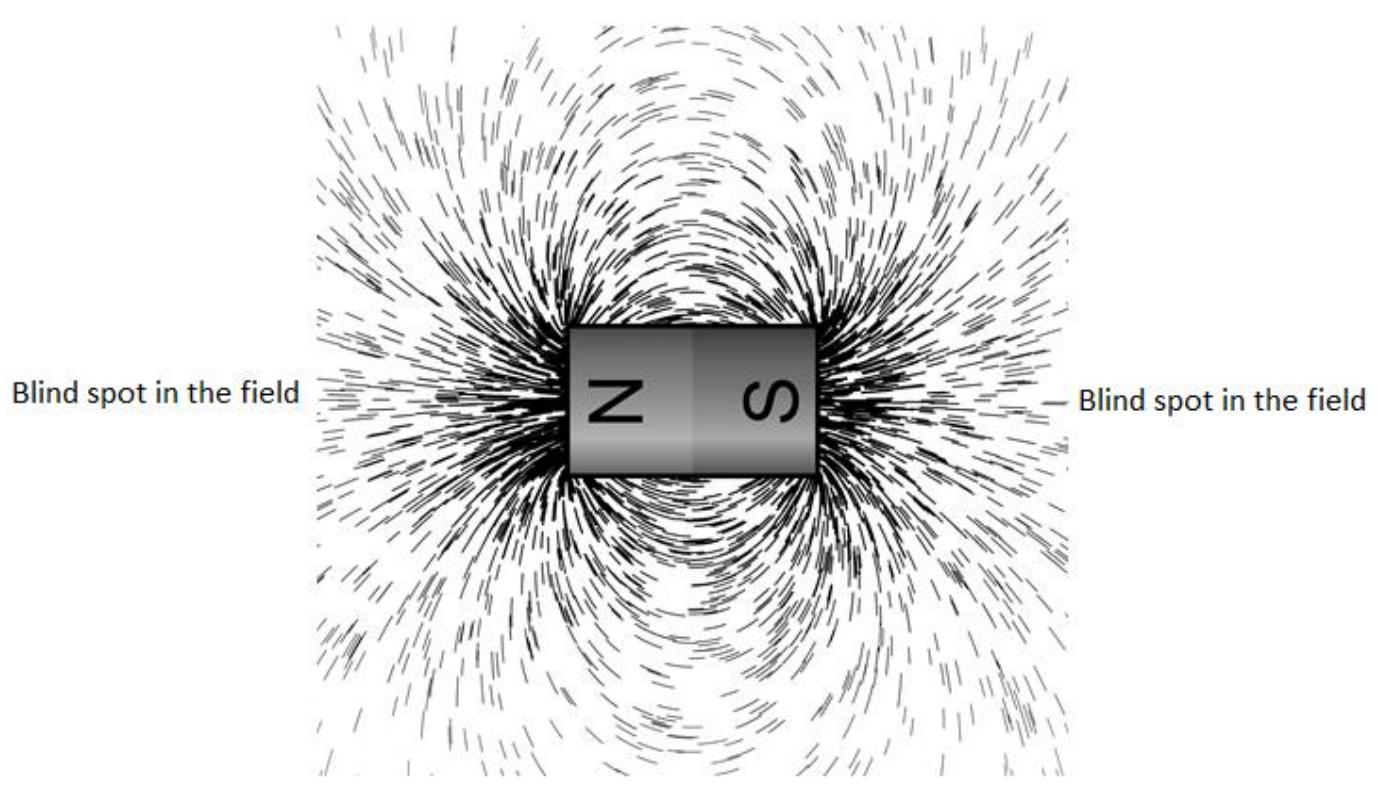

Fig. 2 Shown is a depiction of the magnetic field analogue of the electric field. The

131 drawing shows how iron filings arrange themselves around a magnetic due to the

132 influence of the magnetic field lines. The iron filings effectively map the field lines,

133 showing the blind spot in the field at a macroscopic level, where the field lines in the

134 middle of the far sides of the magnet extend into space with no involvement in the

135 interaction between the magnetic poles.

136 These non-participating field lines exist in a sort of blind spot in the field. This physical

137 condition, by its very nature, affects the coupling of the electrons, as not all field lines

138 are participating in their interaction. It was hypothesized that the blind spot in the

139 electric field affects the numerical value of $\alpha$.

140 If the field surrounding each particle were divided into an odd number of sectors, the 141 non-participating field lines in the blind spot could be relegated to a single region within 
142 the larger field, with an equal portion of the electric field on either side of it. Dividing

143 the field into 3 sectors would be the minimum needed for this purpose, but with this,

144 the blind spot would have to take up nearly a third of the electric field, likely

145 overcompensating for the area. Whatever the best value happens to be, there is also

146 the question of whether it should be considered an ad hoc construct or whether the

147 division is something fundamental to the electric field. Only the latter would be of

148 benefit in understanding the nature of $\alpha$.

149 To help identify an appropriate value to segregate the field lines in the blind spot from

150 the rest of the field, the degrees around the circular field of an unperturbed electron

151 were used. Starting on the far side at the 180-degree mark, in the center of the blind

152 spot, and moving incrementally in 1-degree steps on each side of that mark, fractions of

153360 degrees were identified that resulted in a whole odd number in the denominator

154 (Fig. 3). As shown in Fig. 3, five numbers $(3,5,9,15,45)$ were identified up to a span of

155120 degrees for the whole blind spot (60 degrees on either side of the center).

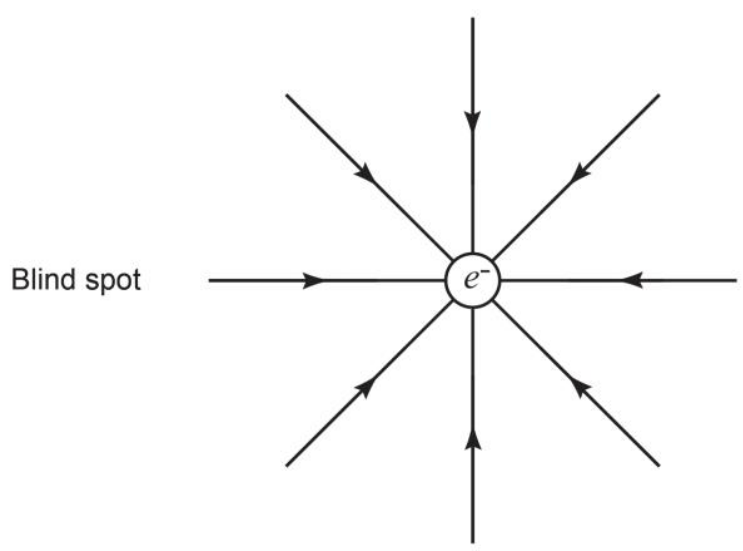




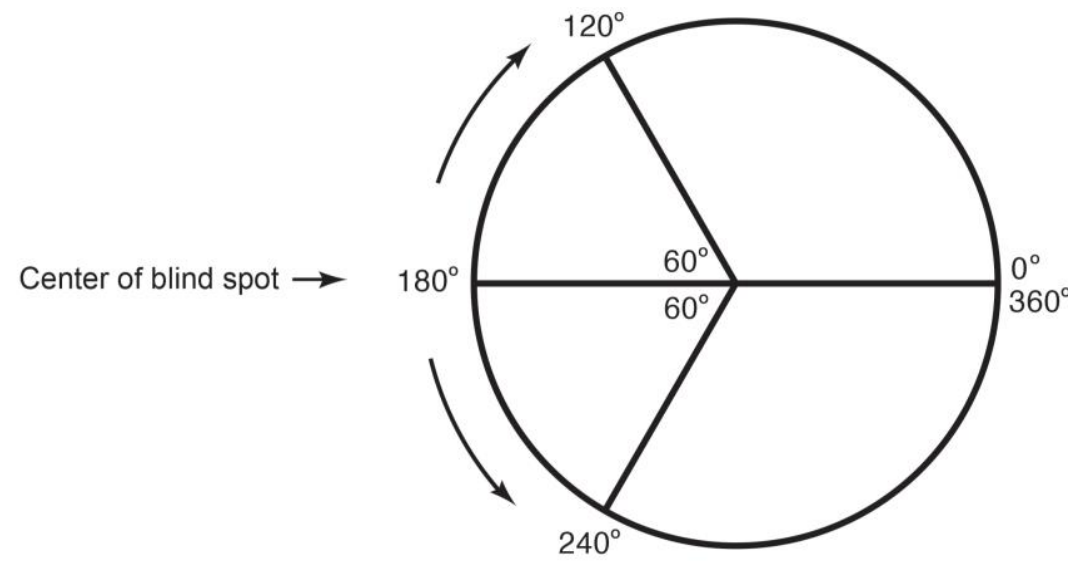

$$
\begin{array}{llllllllllll}
\frac{2}{360} & \frac{4}{360} & \frac{6}{360} & \frac{8}{360} & \cdots & \frac{24}{360} & \cdots & \frac{40}{360} & \cdots & \frac{72}{360} & \cdots & \frac{120}{360}
\end{array}
$$
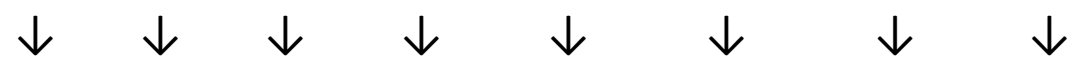

Fig. 3 Division of the electric field into an odd number of sectors, to relegate the blind spot in the field to one sector. Starting on the far side at the 180-degree mark of the field, in the center of the blind spot, and moving incrementally in 1-degree steps on each side of that mark, fractions of $\mathbf{3 6 0}$ degrees were identified that resulted in a whole odd number in the denominator. Five numbers $(3,5,9,15,45)$ were identified up to a span of 120 degrees for the blind spot (60 degrees on either side of the center).

167 A choice of 9 sectors appears, at least superficially, to be appropriate for several 168 reasons: 1) As noted above, a choice of 3 sectors would be the minimum needed but 169 would overcompensate for the blind spot - which would have to take up a third of the 170 field. Nine is the first multiple of 3 within the identified set and leads to a reasonable 171 size for the blind spot, at a little more than a tenth of the field (1/9). 
172 With 5 sectors, the blind spot would have to take up nearly a quarter of the electric

173 field. Thus, similar to 3, it would likely overcompensate for the area. The values of 15

174 and 45 would relegate the blind spot to only about $7 \%$ and $2 \%$ of the field, respectively,

175 likely falling short of the area. Indeed, within the identified set, 9 falls exactly in the

176 middle of the two numbers that would likely overcompensate for the area, and the two

177 that would likely fall short of the area.

178 With the choice of 9 sectors, and one of them representing the blind spot, 8 sectors

179 would be involved in the coupling per particle, Fig. 4.

180
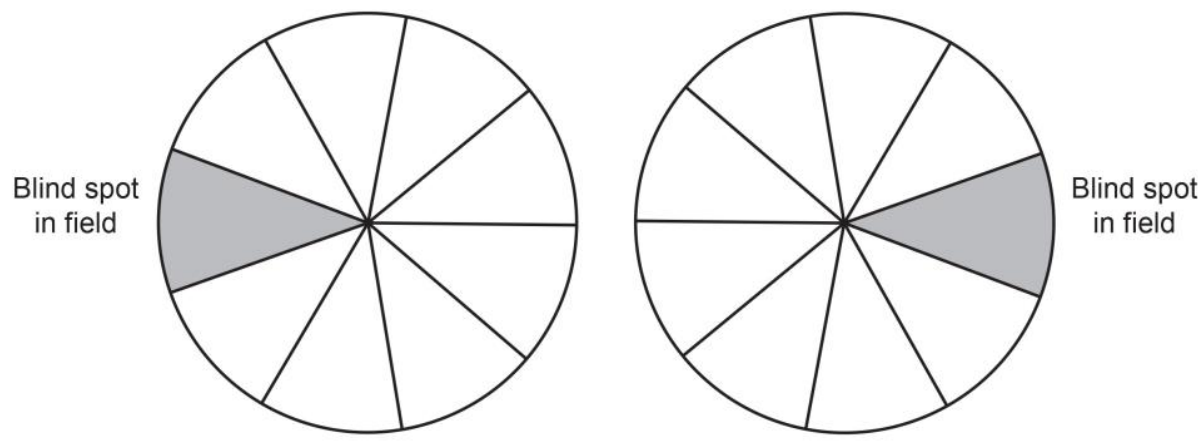

181 Fig. 4|Division of the electron and positron's electric fields into 9 sectors each. Only

182 about 8 sectors per particle would be involved in the coupling. The sector on the far

183 side of each particle represents a blind spot in the field, where the field lines in that

184 sector largely extend into space with no involvement in the interaction.

185 Dividing the ensuing value of 16 from the inverse of the numerical value of $\alpha$ revealed a

186 factor that was approximately the product of Euler's number times pi, leading to the

187 following equation:

$$
\alpha^{-1} \approx(2)(8) e \pi=136.635747562777 \text {. }
$$


189 The fact that two mathematical constants (to an approximation) naturally arose from

190 the choice of 9 (and ultimately 8) sectors per particle also supported the use of 9 for the

191 number of electric field sectors surrounding the particles.

192 However, although the equation produces a result close to the value of $\alpha$, it does not

193 lead to a match with the 2018 CODATA value of the constant ( $\left.\alpha^{-1}=137.035999084[21]\right)$.

194 It is unlikely, however, that the division of the field would be so precise that exactly 8

195 sectors - no more, no less - would be involved in the coupling per particle upon

196 elimination of the blind spot in the field. Rather, it is more likely the case that the sector

197 value would be 8 plus or minus some small amount, likely due, at least in part, to the

198 quantum fluctuations in the overall electromagnetic field, which would alter any

199 geometric precision.

200 Working backward from the currently accepted value of $\alpha$, the electric field sector value

201 for each particle would be approximately 8.02343465913, such that equation (4a) can

202 be recast as follows:

206 The amount in excess of 8 - at 0.0234346591350 - is here referred to as the

207 anomalous electric field sector value, a correction needed on the value of 8 , just as the

208 anomalous magnetic moment value of 0.00231930436182 is needed on the value of 2

209 for $g_{e}$. Indeed, the value of the corrective term needed for the sector value is nearly the

210 same as that needed for $g_{e}$, mostly differing by a simple factor of 10 . Here, the electric 
211 field sector value will be referred to as the "s-factor," $S_{e}$. The proposed general equation

212 for $\alpha$ is thus:

213

$$
\alpha^{-1}=2\left|S_{e}\right| \text { ел }
$$

214 The absolute value is used because in electric repulsion the sectors are, in a sense,

215 "missing" (leading to their having a negative value). In electric repulsion, two electrons

216 (starting off close together) would move apart under the force, filling in the missing

217 sectors of field lines as they separate. In electric attraction, an electron and positron

218 (starting off some distance apart) would move together under the force, eliminating the

219 existing sectors of field lines between them as they unite. $-S_{e}$ can be thought of as an

220 approximately 8-point "hole" to be filled in repulsion, and $S_{e}$ as an approximately 8-

221 point "hill" to be leveled in attraction, to allow full coupling to occur.

222 Note that as the anomalous value for $g_{e}$ is regarded as $2 \alpha_{e}$, where $\alpha_{e}$ is

$2230.00115965218128(18)$, the anomalous value for $S_{e}$ can be regarded as $2\left(S_{e}\right)_{\alpha}$, where

$224\left(S_{e}\right)_{\alpha}$ is 0.0117173295675 . Thus, similar to the equation for $g_{e}$, where $g_{e}=2+2 \alpha_{e}, S_{e}$ can

225 be written as follows:

226

$$
S_{e}=8+2\left(S_{e}\right)_{\alpha}
$$

228 The question remains, however, as to whether $S_{e}$, and thereby equation (5), is actually

229 fundamental in nature, given the apparent ad hoc decision to divide the electric field

230 into 9 sectors to account for the blind spot in the field. It appears that this question can

231 be answered by way of the anomalous value, $\left(S_{e}\right)_{\alpha}$. 
232 As the value of $S_{e}$ is regarded above as a completely arbitrary choice into which to divide

233 the electric field, $\left(S_{e}\right)_{\alpha}$ would be an equally arbitrary value, as it directly stems from that

234 choice. As such, it would be highly improbable for $\left(S_{e}\right)_{\alpha}$ to have any connection to

235 fundamental constants in nature, being much more likely to have nothing to do with

236 them. However, $\left(S_{e}\right)_{\alpha}$ can be derived, to several significant digits, by using the values of

237 the anomalous magnetic moments of the electron, muon, and tau particle $\left(\alpha_{e}, \alpha_{\mu}\right.$, and

$238 \alpha_{\tau}$, respectively). The result is achieved through the following power series:

$$
\frac{\left(S_{e}\right)_{\alpha}}{10} \approx \frac{C_{0}\left(\alpha_{e}+\alpha_{\mu}+\alpha_{\tau}\right)^{0}+C_{1}\left(\alpha_{e}+\alpha_{\mu}+\alpha_{\tau}\right)+C_{2}\left(\alpha_{e}+\alpha_{\mu}+\alpha_{\tau}\right)^{2}+\ldots}{3}
$$

240 where

$242 \alpha_{\mu}=0.00116592061(41)$ (From reference 7)

$243 \quad \alpha_{\tau}=0.001177171(39)$ (From reference 8)

$244 \quad \mathrm{C}_{0}=0$

$245 \quad C_{1}=1$

$246 \quad C_{2}=1+10\left(\alpha_{e}\right)=1.011 \underline{5} 965218128$

$247 \quad C_{3}=1+10\left(\alpha_{\mu}\right)=1.011 \underline{6} 592061$

$248 \quad \mathrm{C}_{4}=1+10\left(\alpha_{\tau}\right)=1.011 \underline{77171}$

$249 \mathrm{C}_{5}=1+$ ??? $\quad \approx 1.011 \underline{8} \ldots$

$250 \quad \mathrm{C}_{6}=1+$ ??? $\quad \approx 1.011 \underline{9} \ldots$

251

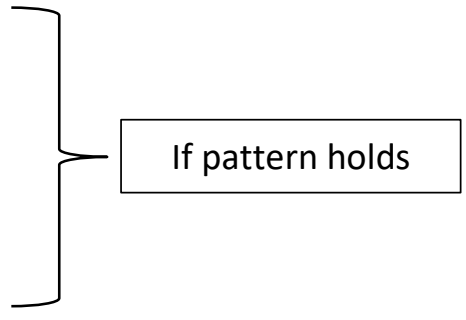


253 Using equation (7) and the values above for $\alpha_{e}, \alpha_{\mu}$, and $\alpha_{\tau}$ leads to an $\left(S_{e}\right)_{\alpha}$ value of

254 about 0.01171733 , compared with 0.0117173295675 from the known value of $\alpha$, a

$25599.99999 \%$ (7-significant-digit) match. Inputting this result into equations (5) and (6)

256 leads to a calculated $\alpha^{-1}$ value of 137.0359 , truncated at the seventh digit due to the low

257 precision level of the $\alpha_{\tau}$ value. Interestingly, an increase in the precision of the value of

$258 \alpha_{\tau}$ through Standard Model calculations is correlated with an increasingly closer match

259 between the value of $\alpha$ as calculated through equations (5), (6), and (7) and the

260 accepted value of the constant (Table 1). It will be interesting to see if increased

261 refinement of the value of $\alpha_{\tau}$ through the Standard Model, or even experimentation,

262 leads to even closer results.

263 Table 1. The increase in the precision of the value of $\alpha_{\tau}$ through Standard Model 264 calculations is correlated with an increasingly closer match between the calculated 265 value of $\alpha$ using the study equations and the accepted value of the constant

\begin{tabular}{|c|c|c|}
\hline Reference & $\begin{array}{l}\text { Value of } \alpha_{\tau} \\
\text { Through } \\
\text { Standard Model } \\
\text { Calculations }\end{array}$ & $\begin{array}{l}\text { Calculated Value of } \alpha^{-1} \text { by Inputting } \\
\text { the } \alpha_{\tau} \text { Value into Equations (5), (6), (7) } \\
\text { in This Study* }\end{array}$ \\
\hline Samuel \& Li, 1991, ref. 9 & $0.0011773(3)$ & $\underline{137.036013882}$ (5 significant digits) \\
\hline $\begin{array}{l}\text { Eidelman \& Passera, 2007, } \\
\text { ref. } 10\end{array}$ & $0.00117721(5)$ & 137.036003561 (6 significant digits) \\
\hline $\begin{array}{l}\text { Keshavarzi, Nomura \& } \\
\text { Teubner, 2020, ref. } 8\end{array}$ & $0.001177171(39)$ & 137.035999089 (7 significant digits) \\
\hline
\end{tabular}

* Significant digits in bold/underline, corresponding to precision of $\alpha_{\tau}$ value.

267 Note: 2018 CODATA value of $\alpha^{-1}=137.035999084(21)$.

268 Equation (7) might not be the only or best equation for showing the relationship

269 between $\alpha_{e}, \alpha_{\mu}, \alpha_{\tau}$ and $\left(S_{e}\right)_{\alpha}$. However, it does so to a notable degree, with the result of 
270 the expression ultimately leading to a good approximation of the value of $\alpha$ that has

271 only increased in precision as the precision of the value of $\alpha_{\tau}$ has increased through

272 Standard Model calculations - with 7 digits of the value of $\alpha$ matched currently.

273 Of course, if future evaluations of $\alpha_{e}, \alpha_{\mu}$, and $\alpha_{\tau}$ lead to an exact match with the value of

$274 \alpha$ through equation (7), this would greatly support the idea of a relationship among the

275 magnetic moment anomalies and $\left(S_{e}\right)_{\alpha}$. However, failure to lead to a result beyond a 7-,

2768 -, or 9-digit match with the value of $\alpha$ could also mean the above equation represents

277 only a limiting case of such a relationship or that an adjustment to the formula is

278 needed. Additional support would come from how well the above information

279 intersects with other areas of physics. For example, as shown in section 2.2 below, the

280 value of $S_{e}$, including its $\left(S_{e}\right)_{\alpha}$ component, can also be formulated in a way that is

281 reflective of the quantum electrodynamics (QED) formula for $g_{e}$, including its $\alpha_{e}$

282 component. This is also the case for $g_{\mu}$ and $g_{\tau}$ and their anomalous components. This

283 moderate intersection with QED further suggests there being some degree of a true

284 mathematical relationship between the magnetic moment anomalies and $\left(S_{e}\right)_{\alpha}$.

285 As a value stemming from the completely arbitrary decision to divide the electric field

286 into 9 sectors, having nothing to do with lepton magnetic moment anomalies, there is

287 no a priori reason for $\left(S_{e}\right)_{\alpha}$ to have any connection with $\alpha_{e}, \alpha_{\mu}$, or $\alpha_{\tau}$, unless there is

288 indeed a preexisting natural relationship among them. Such a link would be highly

289 improbable as a random occurrence, as completely fabricated numbers cannot typically

290 be applied in mathematical analyses in any useful way alongside true physical constants

291 with which they had no original connection. 
292 This suggests that like the values of $\alpha_{e}, \alpha_{\mu}$, and $\alpha_{\tau}$, the value of $\left(S_{e}\right)_{\alpha}$ is indeed a real and 293 natural quantity - an anomalous value associated with the electric field of the electron, 294 muon, and tau particle that is mathematically linked to the anomalous values associated 295 with their magnetic fields, as perhaps another example of how electric and magnetic 296 phenomena are linked. This, in turn, would make the value of $S_{e}$, at approximately 8, 297 also a natural feature of the electric field, and thereby equation (5) a fundamental 298 equation for $\alpha$.

$299 S_{e}$ being a natural value would mean that, similar to the way that the valence shell of 300 atomic nuclei of the main group elements has 8 zones by which the nuclei interact with 301 electrons, there would be 8 zones or sectors in the space surrounding a lepton by which 302 the particle would engage in coupling with another lepton. The main difference is that 303 the lepton, as a single elementary particle, would be interacting with just one other 304 particle, whereas the sectors surrounding an atomic nucleus, which is composed of 305 multiple protons (themselves composed of multiple elementary particles), would be 306 engaged in interactions with multiple (up to 8) electrons. The atom would be in the 307 most energetically stable state when all 8 of the sectors in its valence shell are engaged 308 in interactions with electrons. Similarly, a lepton would be in the most energetically 309 stable state from the standpoint of electromagnetic coupling when all 8 of its field 310 sectors are engaged in the interaction with the other lepton. Thus, there appears to be a 311 certain symmetry between leptons and atoms, with each being surrounded by 8 distinct 312 sectors of space through which interactions occur. 
313 Such spatial organization around an electron is not incompatible with the virtual photon

314 cloud concept. Compagno and colleagues noted that it is possible "...to speak of a cloud

315 of virtual photons [surrounding an electron] having a well defined spatial structure" and

316 that generally it is also possible "...to connect some properties of the spatial structure of

317 clouds to the internal dynamics of the source" [11]. In their study of the cloud of virtual

318 photons associated with the hydrogen atom, Passante and colleagues noted that they

319 were "... led to the conclusion that the structure of the virtual cloud of photons in the

320 ground state is in fact an 'inside-out' mapping of the electronic structure of the

321 hydrogen atom" [12].

322 In contrast to the hydrogen atom, elementary particles such as the electron are

323 considered to be point particles, with no internal structure. However, this is a

324 mathematical abstraction, used in large part because of current technological

325 limitations in probing to sizes that would reveal any internal structure. As such, just as it

326 is suggested that the virtual photon cloud associated with the ground state of the

327 hydrogen atom is an "inside-out" mapping of the electronic structure of the atom, so

328 too might the 8-sector electric field, or equivalent virtual photon cloud, of the electron

329 be an "inside-out" mapping of some finer, as yet identified, electronic structure of the

330 elementary particle. Indeed, the identification of the 8 sectors of the electric field might

331 be a first glimpse into that structure.

332 The question of "Why does nature chose 8 specifically?" cannot be immediately

333 answered for either the lepton or the atom. However, this concept would represent yet

334 another "octet rule" in particle physics: In the model above, a lepton couples with 
335 another lepton through 8 electric field sectors (plus the field's anomalous portion). The

336 "Eightfold Way" concerns the organization of hadrons, and the currently established

337 "Octet Rule" concerns the 8-zoned valence shell of at least the main group elements, 338 even when there are many more electrons between the nucleus and the valence shell.

339 Thus, while there are always exceptions, a "theme of 8" appears to be carried through

340 from leptons, to hadrons, to some lepton-hadron interactions.

341 Although a quantum mechanical analysis would be needed for further evaluation, there

342 are, thus, several clues that the space surrounding an electron does indeed have a

343 discrete, 8-zoned structure, analogous to the valence shell of many atomic nuclei. The

344 most notable support for this concept comes from the fact that the 8-sector model gives

345 rise to the value of $\left(S_{e}\right)_{\alpha}$, which in turn can be derived to several significant digits from

346 the values of the lepton magnetic moment anomalies, which have been established

347 through theory and/or experimentation. Thus, as the magnetic moment anomalies are

348 true physical quantities, so too are likely the values of $\left(S_{e}\right)_{\alpha}$ and $S_{e}$, which directly stem

349 from them.

350 Therefore, $\left(S_{e}\right)_{\alpha}$ and $S_{e}$ appear to be real, nontrivial components in the mathematics of

$351 \alpha$. In the following sections, this is explored further, and it is shown how the concepts of

$352\left(S_{e}\right)_{\alpha}$ and $S_{e}$ help to explain additional electromagnetic phenomenon and provide even

353 greater clarity regarding the mathematical and physical origins of $\alpha$.

\section{$354 \quad 2.1$ Types of $\alpha$ Values}


355 If all 18 sectors were involved between the two interacting particles, $\alpha^{-1}$ would equal

356 about 153 (from 18ел) instead of about 137 (from 16e $\pi$ )-indeed, at a superficial level,

357153 seems as "unusual" a number as 137 . The different scenarios lead to three types of

$358 \alpha$ values, a basic value $\left(\alpha_{B}\right)$ associated with the value 18 , encompassing each interacting

359 particle's full electric field; the true, corrected value of $\alpha$; and a reduced value $\left(\alpha_{R}\right)$ that

360 takes the blind spot per field into account but at a gross level (Table 2).

361 Table 2. Suggested types of the fine-structure constant, $\alpha$, presented as inverse values

\begin{tabular}{|l|l|l|l|}
\hline Type & Symbol & Expression & Value \\
\hline Basic value & $\alpha_{B}{ }^{-1}$ & $18 e \pi$ & 153.715216008 \\
\hline True (corrected) value & $\alpha^{-1}$ & $16.046869325 e \pi$ & $137.035999084^{*}$ \\
\hline Reduced (uncorrected) value & $\alpha_{R}^{-1}$ & $16 e \pi$ & 136.635747562 \\
\hline
\end{tabular}

*2018 CODATA value.

363 The basic value could not really exist, as it would be physically impossible for all 18

364 sectors to be involved in the coupling in any meaningful way. For this to happen, the

365 field would have to be highly contorted to allow the sectors of field lines on the far side

366 of each particle to play a part in the interaction.

367 As the particles move closer to one another under the electric force of attraction, the

368 field lines involved in the interaction would extend over fewer and fewer sectors of

369 space. Ultimately, there would be zero sectors of space between the particles, and the

370 field line density (number of field lines per sector) would become infinite. As a

371 consequence, the coupling strength would become infinite. This is in accordance with 
372 the Landau pole - the state of infinite coupling strength in quantum theory, and in this

373 work is represented as follows:

374

375

376

377

378

379

380

381

382

383

384

385

386

387

388

389

390

391

392

393

394

395

$$
\alpha^{-1}=2|0| \text { ел }=0 ; \alpha=\infty
$$

Thus, the sector value serves as a proxy for field line density, but in the reverse meaning a decrease in the sector value (as an absolute value) corresponds to an increase in field line density:

$$
\text { Field line density }=\frac{\text { Number of field lines }}{\mid \text { Sector value } \mid}
$$

Thus, as noted above, the sectors represent a threshold, or barrier, that must be surmounted for full, or infinite, coupling to occur. The greater the sector value, the more substantial the barrier will be and the lower the field line density, and consequently, the lower the coupling strength.

The sector value concept can also help explain the change in the value of $\alpha$ at rising energy levels when particles are driven together during collider experiments. That is, the value $1 / 137$ is the approximate value of $\alpha$ at low energy. The constant is said to "run," or change, as the particles' energy level changes. When the particles are given higher energy in the collider, the value of $\alpha$ has been measured to rise. At $90 \mathrm{GeV}, \alpha$ has been measured to have a value of approximately $1 / 128.5$ (or 0.007812 ) [13]. This is the identical value attained when one electric field sector (or its equivalent) is lost in 
396

397 398 399

400 electric repulsion, the sector value starts off as negative, and an increase in coupling can

401 be viewed as an increase in this value as the particles move further apart.

$$
\alpha^{-1}=2|8.0234346591350-x| e \pi
$$

$$
\text { (attraction) }
$$

$$
\alpha^{-1}=2|-8.0234346591350+\mathrm{x}| \text { er. }
$$

407 When enough distance is lost in the setting of electric attraction, or gained in the setting 408 of electric repulsion, to fully mitigate the effects of the sector value, the value would go 409 to zero and the strength of the force would reach infinity. Addition or subtraction 410 beyond zero would have no meaning, as you could not remove more sectors than there 411 are to be removed (in electric attraction) or add more sectors around the particles than 412 can be added (in electric repulsion).

\subsection{QED-Like Formula for the Anomalous Electric Field Sector Value}

414 From a geometric perspective, the anomalous electric field sector value can be divided 415 into 2 subsections: a small amount at the upper end of the blind spot in the electric field 
416 and an equally small amount at the lower end, due to a natural symmetry around the

417 circular field (Fig. 5). As the full correction in excess of 8 is 0.0234346591350 , each

418 subsection, $\left(S_{e}\right)_{a}$, would be half of this, or 0.0117173295675 .

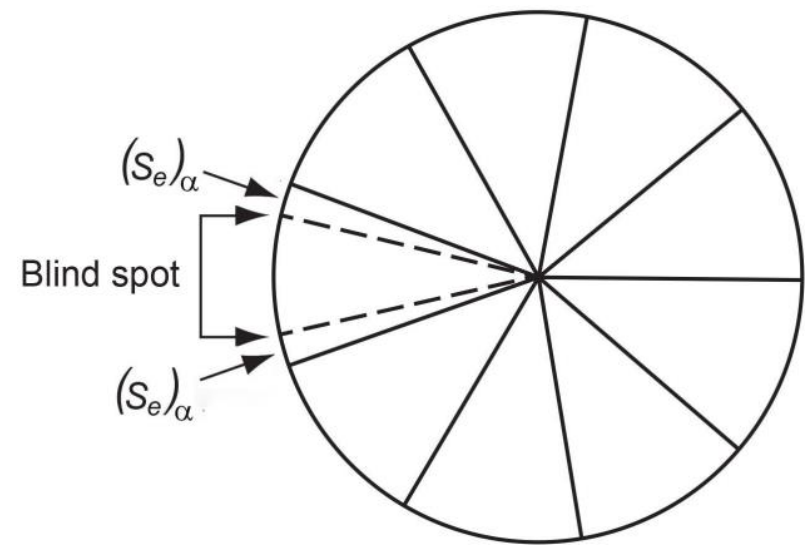

420 Fig. 5 |Shown are the posited 9 sectors of the electric field surrounding an electron.

421 Eight sectors would be involved in an interaction plus a portion of the ninth - a small

422 amount above the blind spot and a small amount below it, corresponding to 2

423 subsections, each here referred to as $\left(S_{e}\right)_{\alpha}$, for the anomalous electric field sector

424 value involved in the interaction. Each subsection would be 0.0117173295675 .

425 Together, they constitute the full correction of 0.0234346591350 needed on the value

4268 in the calculation of $\alpha$.

427 Thus, as noted above, $S_{e}$ can be written as $S_{e}=8+2\left(S_{e}\right)_{\alpha}$, similar to the equation for $g_{e}$,

428 where $g_{e}=2+2 \alpha_{e}$.

429 As with the full value of $g_{e}$, the full value of $S_{e}$ can also be formulated perturbatively. In

430 the case of $g_{e}$, the perturbative method is applied to quantum field theory, specifically

431 QED. The perturbative formula for the QED contribution to $g_{e}$ is as follows: 


$$
\frac{g_{e}}{2}=1+C_{1}\left(\frac{\alpha}{\pi}\right)+C_{2}\left(\frac{\alpha}{\pi}\right)^{2}+C_{3}\left(\frac{\alpha}{\pi}\right)^{3}+C_{4}\left(\frac{\alpha}{\pi}\right)^{4}+\ldots+\text { non }-Q E D
$$

433 The formal power series of $\alpha / \pi$ corresponds to quantum corrections as determined

434 through Feynman diagrams, which in turn correspond to real quantum activity at the

435 particle level. The coefficients of the formula $\left(\mathrm{C}_{\mathrm{i}}\right)$ have been calculated to $(\alpha / \pi)^{5}$ and

436 have been confirmed experimentally [6]:

$$
\mathrm{C}_{1}=0.5 \quad \mathrm{C}_{4}=-1.912 \ldots
$$

The class of quantum activities that would yield a correction on $S_{e}$ is currently not clear.

441 However, $S_{e}$ can be mathematically formulated in a similar way to $g_{e}$ by using $\alpha_{R}$ (again, $4421 / 16$ er, see Table 2 ) in the formal power series in place of $\alpha$ :

$$
\frac{S_{e}}{2}=4+C_{1}\left(\frac{\alpha_{R}}{\pi}\right)+C_{2}\left(\frac{\alpha_{R}}{\pi}\right)^{2}+C_{3}\left(\frac{\alpha_{R}}{\pi}\right)^{3}+C_{4}\left(\frac{\alpha_{R}}{\pi}\right)^{4}+C_{5}\left(\frac{\alpha_{R}}{\pi}\right)^{5}
$$

444 Using mathematical deduction, several sets of coefficients for equation (13) were

445 identified, each leading to approximately the same value for $\alpha$ when the corresponding

$446 S_{e}$ value was inserted into equation (5). Each solution is also consistent with the 2018

447 CODATA value of the constant (Table 3). 
448 In each set, the base values of $C_{1}$ though $C_{5}$ are the same, at $0.5,4 / \pi, 3 / \pi, 2 / \pi$, and $1 / \pi$,

449 respectively, largely following a pattern as multiples of $1 / p i$. $C_{1}$ and $C_{2}$ in each set have

450 exponents of 1 . Exponents for $C_{3}$ through $C_{5}$ begin with a value of 3 in each set. Starting

451 at $C_{3}$, the exponents follow a simple numerical sequence in set 1 , the prime number

452 sequence in set 2 (which actually can be regarded as beginning with $C_{2}$ ), multiples of 3 in

453 set 3 , and every fourth number in set 4 . Note that each coefficient must be multiplied by

454 a factor of 10 before applying it to equation (13).

455 Table 3. Coefficients for Equation (13), Four Possible Sets*

\begin{tabular}{|l|l|l|l|l|}
\hline Coefficient & Set 1 & Set 2 & Set 3 & Set 4 \\
\hline$C_{1}$ & 0.5 & 0.5 & 0.5 & 0.5 \\
\hline$C_{2}$ & $4 / \pi$ & $4 / \pi$ & $4 / \pi$ & $4 / \pi$ \\
\hline$C_{3}$ & $(3 / \pi)^{3}$ & $(3 / \pi)^{3}$ & $(3 / \pi)^{3}$ & $(3 / \pi)^{3}$ \\
\hline$C_{4}$ & $(2 / \pi)^{4}$ & $(2 / \pi)^{5}$ & $(2 / \pi)^{6}$ & $(2 / \pi)^{7}$ \\
\hline$C_{5}$ & $(1 / \pi)^{5}$ & $(1 / \pi)^{7}$ & $(1 / \pi)^{9}$ & $(1 / \pi)^{11}$ \\
\hline $\begin{array}{l}\text { Calculated } \\
\alpha^{-1} \text { value }\end{array}$ & $137.035999085306 \ldots$ & $137.035999084705 \ldots$ & $137.035999084323 \ldots$ & $137.035999084080 \ldots$ \\
\hline
\end{tabular}

457 *Each coefficient in the table must be multiplied by a factor of 10 before applying to 458 equation (13).

459

460 In the above case, the factor of 10 is considered to be part of the coefficient. Setting 10

461 apart from the coefficients, the equation can be written as follows: 


$$
\frac{S_{e}}{2}=4+(10)\left(C_{1}\left(\frac{\alpha_{R}}{\pi}\right)+C_{2}\left(\frac{\alpha_{R}}{\pi}\right)^{2}+C_{3}\left(\frac{\alpha_{R}}{\pi}\right)^{3}+C_{4}\left(\frac{\alpha_{R}}{\pi}\right)^{4}+C_{5}\left(\frac{\alpha_{R}}{\pi}\right)^{5}\right)
$$

464 Sets 3 and 4 each appear to lead to the closest match with the current CODATA value,

465 excluding its margins of error.

466 A recent experiment, producing the most precise measurement of $\alpha$ as of 2020,

467 suggests that $\alpha^{-1}$ might have a value closer to $137.035999206(11)$ [14]. In this setting,

468 the coefficients could be similar to those listed below, still with a pattern among the 469 values:

$$
C_{1}=0.5
$$

$$
\mathrm{C}_{2}=\frac{4}{\pi}
$$

$$
C_{4}=\frac{\sqrt[4]{12}}{\pi}
$$

474

475

476

$$
C_{3}=\frac{\sqrt{8}}{\pi}
$$

477 These coefficients would lead to an $\alpha^{-1}$ value of 137.035999217 , at the upper end of the 478 experimental value range. 
479 The perturbative treatments of $S_{e}$ above might serve as purely mathematical formulas.

480 However, given the similarities of the perturbative formulations for $g_{e}$ and $S_{e}$ and the

481 similarity of the anomalous values - at 0.00231930436 for $g_{e}$ and 0.023434659 for $S_{e}-$

482 the perturbative formulations for $S_{e}$ might be associated with actual physical

483 phenomena at the quantum level, just as the perturbative formulation for $g_{e}$ is. Indeed,

484 as shown above in equation (7), the value of $\left(S_{e}\right)_{\alpha}$ appears derivable by using the values

485 of $\alpha_{e}, \alpha_{\mu}$, and $\alpha_{\tau}$, which are themselves derivable through QED. This too supports the

486 idea of $\left(S_{e}\right)_{\alpha}$, and thereby of $S_{e}$, being associated with actual quantum activity.

487 Note that the ability to identify coefficients by mathematical deduction does not

488 preclude the role of quantum mechanics in nature. For example, the following

489 coefficients, determined by mathematical deduction, could be used in equation (12) for

490 calculating $g_{e}$ :

$$
C_{1}=0.5
$$

$$
C_{2}=-\frac{1}{3}
$$

$$
C_{3}=\frac{\pi^{2}}{3}
$$

$$
C_{4}=-\pi^{2}
$$

$$
C_{5}=2 \pi^{2} .
$$


497 They lead to a $g_{e}$ value of 2.00231930436249 , a $99.99999999999 \%$ (13-significant-digit) 498 match with the 2018 CODATA value of 2.00231930436256 (35). QED, however, is one of 499 the most verified physical theories, indicating the existence of actual quantum activity.

500 A better understanding of the quantum activity leading to the anomalous component of $501 S_{e}$ is needed, perhaps leading to one of the above perturbative formulas or a different 502 one. However, the presented mathematical work does demonstrate that such a 503 formula, linked to actual quantum mechanical phenomena, might be possible.

504 Whether or not a quantum mechanical basis to $\alpha$ is found, it is clear that the constant 505 can be well formulated by using mathematical constants in contrast to what is generally 506 believed today. One of the notable aspects of the formula is its self-consistent nature 507 that is, $\alpha_{R}$ is the number to be corrected and is, at the same time, the tool by which the 508 correction is attained, as the numerator of the parameter in the power series.

509 Caution should be exercised, however, in attempting to mathematically model whatever 510 the latest CODATA value happens to be. The CODATA value is simply determined

511 through the best experimental studies available at the time it is established, and 512 experimental values have a tendency to shift slightly. Whereas the 2018 CODATA value 513 for $\alpha^{-1}$ is $137.035999084(21)$, the 2014 CODATA value was 137.035999139(31), which 514 would require a different set of coefficients than those above.

515 Also, while the above perturbative formulas are characterized by particular patterns 516 among the coefficients, a valid formula for a different experimental value for $\alpha$ might 
517 not have a pattern (or at least not an obvious one). The principal idea here is that

518 flexibility in any mathematical model is important.

519 If an obvious pattern is detected among the coefficients, and experimental results

520 consistently agree with such a pattern, there is also the possibility that the formula

521 could be used to calculate $\alpha$ to an indefinite number of decimal places, if the expansion

522 diverges. The convergence of such a series would, of course, be invaluable information,

523 as well.

524 In all, the perturbative treatment for $S_{e}$ shown above in conjunction with equation (5)

525 represents, for the first time in history, a full mathematical expression for $\alpha$ that

526 involves mathematical constants, leads to an exact match with the established value of

527 the constant, has the potential to calculate $\alpha$ to an indefinite number of decimal places,

528 and is linked to a physical aspect of the electric field. This physical aspect concerns field

529 geometry at a gross level (accounting for a blind spot in the field), but likely also

530 quantum activity within the greater electromagnetic field.

5312.3 Alternate Formulas for the Anomalous Electric Field Sector Value

532 As alternate mathematical expressions for calculating a quantity can often be

533 informative, the perturbative solution above was converted into other forms: 1) an

534 alternate perturbative series, with a leading term slightly higher than the value of 4 used

535 above, 2) a related expansion series with non-integer exponents, and 3) a generalized

536 continued fraction. Each offers additional insight into $\alpha$ that might prove useful in

537 further analysis of the constant, both from a mathematical and physical perspective. 
539 In addition to the above, the full value of $S_{e}$ can be formulated as follows:

540

$$
\frac{S_{e}}{2}=C_{0} \alpha_{R}^{0}+C_{1} \alpha_{R}+C_{2} \alpha_{R}^{2}+C_{3} \alpha_{R}^{3}+C_{4} \alpha_{R}^{4}+C_{5} \alpha_{R}^{5}+\cdots
$$

541 where again, $\alpha_{R}$ is the reduced fine-structure constant (1/16e $\left.\pi\right)$. Given the currently

542 accepted value of $\alpha$, the following values appear appropriate for the coefficients:

543

544

545

546

547

548 Unlike the expression in the previous section, the exactly solvable portion of this

549 expression - that is, the leading term represented by coefficient, $\mathrm{C}_{0}-$ is 4 plus a small

550 amount from an expression involving pi. Such a formula could be important if there is a

551 geometrical aspect to the anomalous value (which the expression after 4 in $\mathrm{C}_{0}$ would

552 likely account for) in addition to quantum activity (which the first through the fifth terms

553 would account for).

554 From $\mathrm{C}_{3}$ onward, the coefficients appear to be a fraction where each numerator is pi and

555 each denominator falls within a linear sequence starting with 3 and then every fourth

556 number thereafter (i.e., 3, 7, 11, ...). Increased precision on the 2018 CODATA value

557 would be needed to determine if the pattern remained from $C_{5}$ onward. When applied

558 to equation (5), the expression leads to a value for $\alpha^{-1}$ of 137.035999084, an exact, 12-

559 significant-digit match with the 2018 CODATA value. 

exponents:

where the coefficients are as follows:

$$
\begin{gathered}
\frac{S_{e}}{2}=C_{0} \alpha_{R}^{0}+C_{1} \alpha_{R}+C_{2} \alpha_{R}^{2}+C_{3} \alpha_{R}^{3}+C_{4} \alpha_{R}^{4}+ \\
C_{5} \alpha_{R}^{4.001}+C_{6} \alpha_{R}^{4.002}+C_{7} \alpha_{R}^{4.003}+ \\
C_{8} \alpha_{R}^{4.004}+C_{9} \alpha_{R}^{4.005}+C_{10} \alpha_{R}^{4.006} \ldots,
\end{gathered}
$$

570 There is a certain "elegant simplicity" to this formula, where all of the coefficients from

$571 C_{2}$ onward are 1. The 5th through the 10th terms, with exponents of 4.001 through

5724.006 , appear as a subset of the 4th term, with the leading term to the 4th term being

573 the principal portion of the equation.

\section{$574 \quad$ 2.3.2. Generalized Continued Fraction}

575 Fig. 6 shows the full value of $S_{e}$ modeled as a generalized continued fraction. The

576 number 8 (for the number of principal electric field sectors involved in the coupling) is

577 the leading term of the fraction. The remaining component represents the anomalous

578 value. The partial numerators of the fraction begin with the number 3 and are 1

579 thereafter. The partial denominators comprise two distinct areas within the fraction:

580 one representing a major set of corrections, with the values of $2^{7}$ and $2^{6}$, in that order, 581 and one representing a minor set, with the values of 2, 4, and 6, also in that order. The 
582 major set of corrections alone is associated with an $\alpha^{-1}$ value of 137.035998746, a

583 99.999999\% (8-significant-digit) match with the currently accepted value. The full

584 fraction, of course, leads to the exact value of $\alpha$.

585

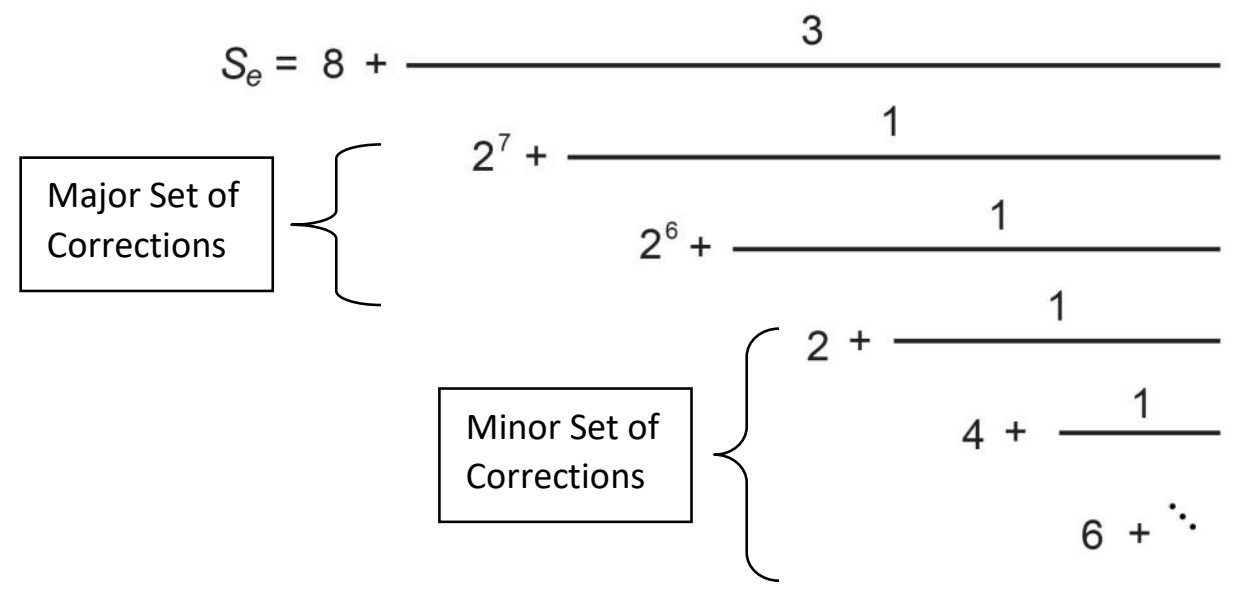

$586 \quad \alpha^{-1}=2\left|S_{e}\right| e \pi$

$587 \quad \alpha^{-1}=137.035999084$ (via formula)

$588 \quad \alpha^{-1}=137.035999084(21)($ CODATA, 2018)

589 Exact match.

590 Fig. 6|Shown is the value of $S_{e}$, including its anomalous component, modeled as a

591 generalized continued fraction. The partial denominators comprise two distinct areas

592 within the fraction (which is truncated in the figure): one representing a major set of

593 corrections, with the values of $2^{7}$ and $2^{6}$, in that order, and one representing a minor

594 set, with the values of $\mathbf{2 , 4}$, and 6 , also in that order. The fraction, in conjunction with

595 equation (5), fully models the currently accepted value of $\alpha$ mathematically to an

596 exact match. 
597 There are several noteworthy issues concerning the fraction, the first of which is its

598 regular structure, providing a straightforward representation of the full value of $S_{e}$.

599 Indeed, the possibility exists that the fraction has a regular structure that extends

600 indefinitely, as the generalized continued fraction of pi does, although a more precise

601 experimental value of $\alpha$ would be needed to be sure.

602 For example, the partial denominators could continue as multiples of 2 or even powers

603 of 2. Using multiples of 2 and extending the fraction after the partial denominator of 6

604 through a partial denominator of 12 results in an $\alpha^{-1}$ value of 137.035999084059 .

605 Extending the partial denominators as powers of 2, going from 2 through $2^{6}$, results in

606 an $\alpha^{-1}$ value of 137.035999083716 . Both are consistent with 2018 CODATA value of

607 137.035999084(21) (Fig. 7).

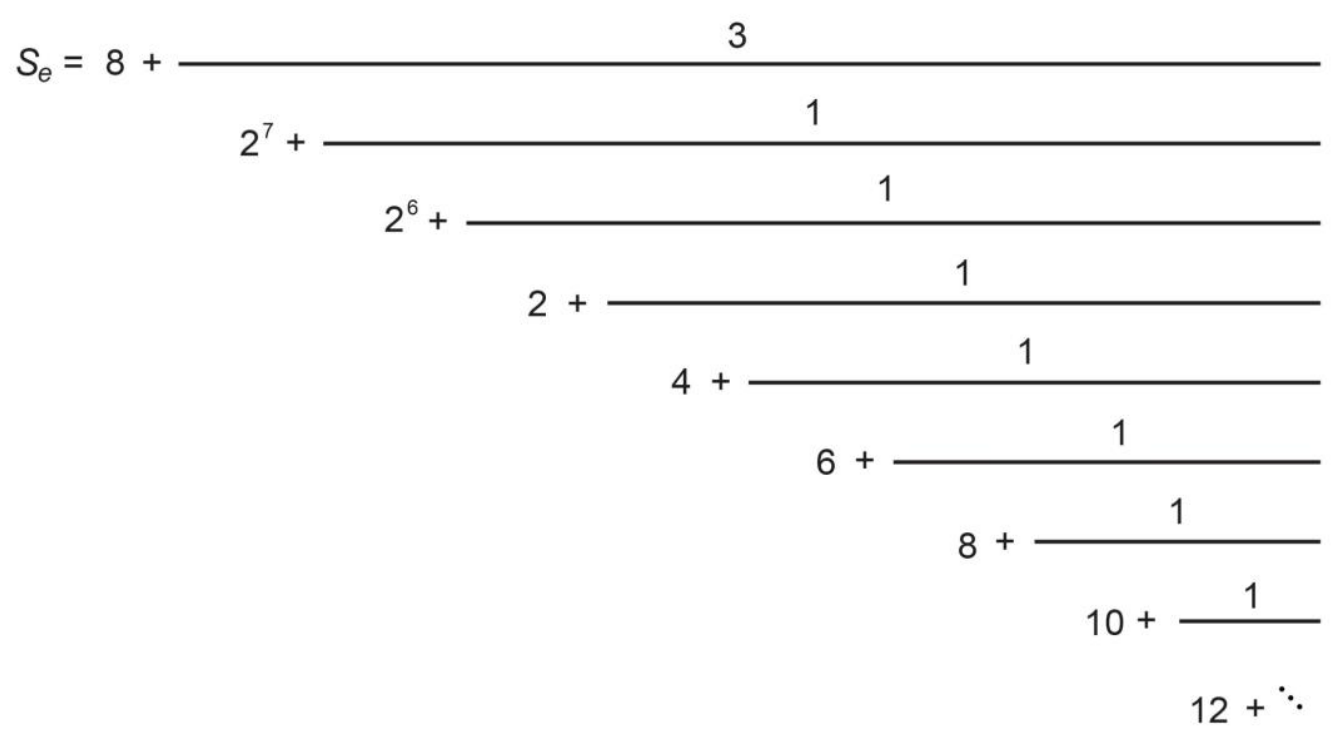

609 Extending partial denominators as multiples of 2 leads to an $\alpha^{-1}$ value of

$610 \quad 137.035999084059 . .$.

611 


$$
\begin{aligned}
& S_{e}=8+\frac{3}{2^{7}+\frac{1}{2}} \\
& 2^{7}+\frac{1}{2^{6}+\frac{1}{}} \\
& 2^{6}+\frac{1}{1} \\
& 2+\frac{1}{2^{2}+\frac{1}{1}} \\
& 2^{3}+\frac{1}{-} \\
& 2^{4}+\frac{1}{-} \\
& 2^{5}+\frac{1}{-1}
\end{aligned}
$$

613 Extending partial denominators as powers of 2 leads to an $\alpha^{-1}$ value of $614 \quad 137.035999083716 \ldots$

$$
\pi=3+\frac{1^{2}}{6+\frac{3^{2}}{6+\frac{5^{2}}{6+\frac{7^{2}}{6+\ddots}}}}
$$

617 Pi shown as a generalized continued fraction with a regular structure that extends

618 indefinitely, for comparison.

619 Fig. 7|Possible generalized continued fractions for $S_{e}$, each with a regular structure.

620 Two examples are shown: A) one where the lower partial denominators continue as

621 multiples of 2, and B) one where they continue as powers of 2. Each example leads to 
622 a value for $\alpha$ that is consistent with the currently accepted value of the constant. C)

623 The regular structure of a generalized continued fraction for pi is shown for

624 comparison.

625 The generalized continued fraction for $S_{e}$ would be a particularly powerful tool if future

626 evidence suggests that it does indeed have a regular structure throughout. If so, the

627 fraction could be used to determine the value of $\alpha$ to as many decimal places as desired,

628 similar to the potential of the perturbative formulas above. Of course, the possibility

629 also exists that there is no regular structure, or not one beyond the partial

630 denominators of $2^{7}$ and $2^{6}$. There is the further possibility that the fraction is finite, with

631 or without a regular structure.

632 As noted above, a recent experiment suggests $\alpha^{-1}$ might have a value closer to

$633137.035999206(11)$ [14]. This would be consistent with a generalized continued fraction

634 where all partial numerators and denominators following the partial denominator of $2^{6}$

635 were a 1 , or simply where the remaining fraction after $2^{6}$ were the inverse of the Golden

636 Ratio, 1.6180339887498948482. In this case, $\alpha^{-1}$ calculates to a value of approximately

637 137.035999213, which falls within the margin of error of the experimental result (Fig. 8). 


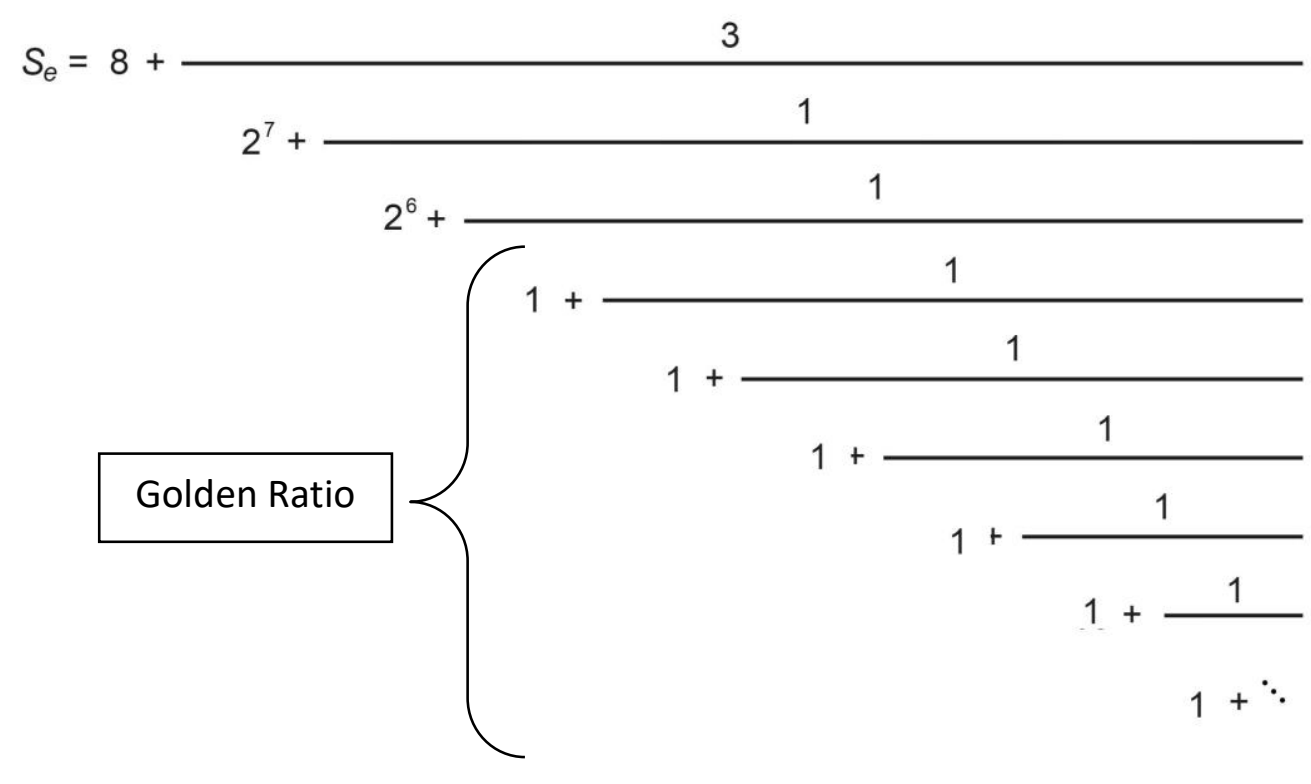

639 Fraction leads to an $\alpha^{-1}$ value of 137.035999213 , consistent with the recent

640 experimental result of $137.035999206(11)[14]$.

641 Fig. 8|A generalized continued fraction for $S_{e}$, where the partial numerators and

642 denominators following the partial denominator of $2^{6}$ all have the value of 1 , leading

643 to an $\alpha^{-1}$ value of approximately 137.035999213 , consistent with the recent

644 experimental result of $137.035999206(11)$ [14]. In this case, the remaining fraction

645 after the partial denominator of $2^{6}$ would simply be the inverse of the Golden Ratio.

646 Another noteworthy aspect of the expression is the fact that there are large terms early

647 in the fraction (again, $2^{7}$ and $2^{6}$ ), making the anomalous value easy to approximate

648 through rational values and leading to a substantial match with the established value of

$649 \alpha$ by way of only a few mathematical steps. Indeed, normal rounding procedures lead to

650 a 9-significant-digit match (137.035998746 to 137.035999$)$. As such, these two partial

651 denominators are possibly a firm, universal truth concerning the (zero energy) value of 
652 the constant. That is, as noted above, experimental results have a tendency to differ

653 slightly. As such, the terms of the fraction that would follow $2^{6}$ cannot yet be definitively

654 stated. However, many, if not all, agree on at least the first 9 significant digits of the

655 constant's value, which the first two partial denominators of the generalized continued

656 fraction well lead to.

657 The portion after $2^{6}$ could be an irrational value, such as the following:

$$
S_{e}=8+
$$
3

661 Although not a traditional generalized continued fraction, this expression also leads to a 662 value for $\alpha$ that is a 12-significant-digit (exact) match with the current CODATA value of 663 the constant. The point here is simply that something "nonclassical" might be happening 664 at a physical level in relation to the area of the fraction following $2^{6}$ (or a lower point), 665 likely quantum mechanical activity. This would be consistent with the fact that many 666 experiments agree on the first 9 significant digits of $\alpha$, which again, the portion of the 667 fraction down to $2^{6}$ will lead to. However, they tend to differ slightly beyond this point. 668 A robust quantum mechanical analysis, if possible, might help to definitively home in on 669 the value of $\alpha$ beyond the first 9 significant digits, and thereby this area of the fraction 670 and which experimental results are likely the most accurate.

\section{3. Conclusion}


672 The nature of $\alpha$ has been a mystery since its discovery more than 100 years ago, and

673 there have been numerous attempts to identify the mathematical basis of the constant.

674 This study presents a full mathematical formula for $\alpha$ that leads to an exact match with

675 the 2018 CODATA value of the constant, and that, importantly, is connected to a

676 physical aspect of the electric field. As such, it is likely connected to quantum

677 mechanical activity also, as the overall electromagnetic field is quantized in nature.

678 At the heart of the mathematics is the idea of a dimensionless anomalous electric field

679 value of about 0.023 associated with the electron. This is particularly notable, as the

680 electron is also associated with a dimensionless anomalous magnetic field value of

681 about $0.023 / 10$, which has been well established through perturbative methods applied

682 to QED and through experimentation. In fact, the anomalous value for the electric field

683 is mathematically linked to the anomalous values of the magnetic fields of each of the

684 charged leptons by way of a simple expression, suggesting that it is a real feature of the

685 electric field, and further suggesting yet another link between electric and magnetic

686 phenomena.

687 Altogether, the concepts introduced here suggest that $\alpha$ is not a random value in the

688 universe, nor does it represent an impenetrable box. Instead, there appear to be

689 accessible mathematics and physics (i.e., quantum activity) inside this box governing the

690 constant and thereby all of the physical phenomena associated with it. This suggests

691 that deeper levels of understanding and discovery might be possible in the setting of the

692 constant. And as $\alpha$ is one of about two dozen dimensionless constants upon which the

693 universe is built, it also suggests that it might be possible to simplify the universe into a 
694 small set of mathematical constants. The constants might simply need to be arranged in 695 different ways in equations in association with certain physical (perhaps geometrical) 696 conditions of particles and fields, with quantum mechanical activity "filling in the gaps" 697 in terms of attaining accurate and precise results.

698 Of particular note, the transformation of $\alpha$ from an ad hoc value in the Standard Model 699 would serve as a sizeable crack in the ceiling holding the Standard Model back from 700 being a more complete theory of the elementary particles and their interactions. And as $701 \alpha$ unites fundamental aspects of electromagnetism, quantum physics, and relativity, a 702 deeper understanding of its nature might also assist in efforts to unite the seemingly 703 incompatible physical theories of general relativity and quantum mechanics.

\section{4. References}

705 1. Tiesinga, E., Mohr, P.J., Newell, D.B. \& Taylor, B.N. The 2018 CODATA recommended values of the fundamental physical constants (web version 8.1). Database developed by Baker, J., Douma, M. \& Kotochigova, S. Available at http://physics.nist.gov/constants, National Institute of Standards and Technology, Gaithersburg, MD 20899 (2020).

2. Dirac, P. A. M. The quantum theory of the electron. Proc. R. Soc. Lond. A 117 (778), 610-624 (1928).

3. Aoyama, T., Hayakawa, M., Kinoshita, T. \& Nio, M. Tenth-order QED contribution to the electron $g-2$ and an improved value of the fine structure constant. Phys. Rev. Lett. 109, 111807 (2012). 
4. Aoyama, T., Hayakawa, M., Kinoshita, T. \& Nio, M. Tenth-order electron anomalous magnetic moment - contribution of diagrams without closed lepton loops. Phys. Rev. D 91, 033006 (2015).

5. Aoyama, T., Kinoshita, T. \& Nio, M. Revised and improved value of the QED tenth-order electron anomalous magnetic moment. Phy. Rev. D 97, 036001 (2018).

6. Aoyama, T., Kinoshita, T. \& Nio, M. Theory of the anomalous magnetic moment of the electron. Atoms 7, 28 (2019).

7. Abi, B. et al. (Muon g-2 collaboration). Measurement of the positive muon anomalous magnetic moment to 0.46 ppm. Phy. Rev. Lett. 126, 141801 (2021).

8. Keshavarzi, A., Nomura, D. \& Teubner, T. g-2 of charged leptons, $\alpha\left(M \frac{2}{Z}\right)$ and the hyperfine splitting of muonium. Phys. Rev. D 101, 014029 (2020).

9. Samuel, M.A. \& Li, G. Anomalous magnetic moment of the tau lepton. Phy. Rev. Lett. 67, 668-670 (1991).

10. Eidelman, S. \& Passera, M. Theory of the tau lepton anomalous magnetic moment. Mod. Phys. Lett. A 22, 159-179 (2007).

11. Compagno, G., Passante, R., Persico, F. \& Salamone, G.M. Cloud of virtual state of the hydrogen atom. Phys. Rev. A 31, 2827-2840 (1985). 
743 manuscript.

\section{4}

745

750 article.

751 The author certifies that he has no affiliations with or involvement in any organization or

752 entity with any financial interest or non-financial interest in the subject matter or

753 materials discussed in this manuscript.

754 The author has no financial or proprietary interests in any material discussed in this

755 article.

756 The data that support the findings of this study are openly available in the 2018 CODATA

757 Recommended Values of the Fundamental Physical Constants, reference number 1. 


\section{8. Figure Legends}

759 Fig. 1|The electric interaction between an electron and positron (before annihilation), 760 as depicted using classical field lines. On the far side of the particles, there are field lines 761 that do not take part in the interaction, with the field lines simply extending into space, 762 representing a blind spot in the field.

763 Fig. 2 Shown is a depiction of the magnetic field analogue of the electric field. The 764 drawing shows how iron filings arrange themselves around a magnetic due to the 765 influence of the magnetic field lines. The iron filings effectively map the field lines, 766 showing the blind spot in the field at a macroscopic level, where the field lines in the 767 middle of the far sides of the magnet extend into space with no involvement in the 768 interaction between the magnetic poles.

769 Fig. 3|Division of the electric field into an odd number of sectors, to relegate the blind

770 spot in the field to one sector. Starting on the far side at the 180-degree mark of the

771 field, in the center of the blind spot, and moving incrementally in 1-degree steps on

772 each side of that mark, fractions of 360 degrees were identified that resulted in a whole

773 odd number in the denominator. Five numbers $(3,5,9,15,45)$ were identified up to a

774 span of 120 degrees for the blind spot (60 degrees on either side of the center).

775 Fig. 4|Division of the electron and positron's electric fields into 9 sectors each. Only 776 about 8 sectors per particle would be involved in the coupling. The sector on the far side 777 of each particle represents a blind spot in the field, where the field lines in that sector 778 largely extend into space with no involvement in the interaction. 
779 Fig. 5|Shown are the posited 9 sectors of the electric field surrounding an electron.

780 Eight sectors would be involved in an interaction plus a portion of the ninth - a small

781 amount above the blind spot and a small amount below it, corresponding to 2

782 subsections, each here referred to as $\left(S_{e}\right)_{\alpha}$, for the anomalous electric field sector value

783 involved in the interaction. Each subsection would be 0.0117173295675 . Together, they

784 constitute the full correction of 0.0234346591350 needed on the value 8 in the

785 calculation of $\alpha$.

786 Fig. 6 Shown is the value of $S_{e}$, including its anomalous component, modeled as a

787 generalized continued fraction. The partial denominators comprise two distinct areas

788 within the fraction (which is truncated in the figure): one representing a major set of

789 corrections, with the values of $2^{7}$ and $2^{6}$, in that order, and one representing a minor

790 set, with the values of 2, 4, and 6, also in that order. The fraction, in conjunction with

791 equation (5), fully models the currently accepted value of $\alpha$ mathematically to an exact

792 match.

793 Fig. 7|Possible generalized continued fractions for $S_{e}$, each with a regular structure.

794 Two examples are shown: A) one where the lower partial denominators continue as

795 multiples of 2, and B) one where they continue as powers of 2. Each example leads to a

796 value for $\alpha$ that is consistent with the currently accepted value of the constant. C) The

797 regular structure of a generalized continued fraction for pi is shown for comparison.

798 Fig. 8|A generalized continued fraction for $S_{e}$, where the partial numerators and

799 denominators following the partial denominator of $2^{6}$ all have the value of 1 , leading to 

result of $137.035999206(11)$ [14]. In this case, the remaining fraction after the partial denominator of $2^{6}$ would simply be the inverse of the Golden Ratio.

Table 1. The increase in the precision of the value of $\alpha_{\tau}$ through Standard Model calculations is correlated with an increasingly closer match between the calculated value of $\alpha$ using the study equations and the accepted value of the constant

\begin{tabular}{|c|c|c|}
\hline Reference & $\begin{array}{l}\text { Value of } \alpha_{\tau} \\
\text { Through } \\
\text { Standard Model } \\
\text { Calculations }\end{array}$ & $\begin{array}{l}\text { Calculated Value of } \alpha^{-1} \text { by Inputting } \\
\text { the } \alpha_{\tau} \text { Value into Equations (5), (6), (7) } \\
\text { in This Study* }\end{array}$ \\
\hline Samuel \& Li, 1991, ref. 9 & $0.0011773(3)$ & 137.036013882 (5 significant digits) \\
\hline $\begin{array}{l}\text { Eidelman \& Passera, 2007, } \\
\text { ref. } 10\end{array}$ & $0.00117721(5)$ & 137.036003561 (6 significant digits) \\
\hline $\begin{array}{l}\text { Keshavarzi, Nomura \& } \\
\text { Teubner, 2020, ref. } 8\end{array}$ & $0.001177171(39)$ & 137.035999089 (7 significant digits) \\
\hline
\end{tabular}

* Significant digits in bold/underline, corresponding to precision of $\alpha_{\tau}$ value.

808 Note: 2018 CODATA value of $\alpha^{-1}=137.035999084(21)$.

Table 2. Suggested types of the fine-structure constant, $\alpha$, presented as inverse values

\begin{tabular}{|l|l|l|l|}
\hline Type & Symbol & Equation & Value \\
\hline Basic value & $\alpha_{B}^{-1}$ & $18 \mathrm{e} \pi$ & 153.715216008 \\
\hline True (Corrected) value & $\alpha^{-1}$ & $16.046869325 \mathrm{e} \pi$ & $137.035999084^{*}$ \\
\hline Reduced (Uncorrected) value & $\alpha_{R}^{-1}$ & $16 \mathrm{e} \pi$ & 136.635747562 \\
\hline
\end{tabular}

*2018 CODATA value. 
812 Table 3. Coefficients for Equation (13), Four Possible Sets*

\begin{tabular}{|l|l|l|l|l|}
\hline Coefficient & Set 1 & Set 2 & Set 3 & Set 4 \\
\hline$C_{1}$ & 0.5 & 0.5 & 0.5 & 0.5 \\
\hline$C_{2}$ & $4 / \pi$ & $4 / \pi$ & $4 / \pi$ & $4 / \pi$ \\
\hline$C_{3}$ & $(3 / \pi)^{3}$ & $(3 / \pi)^{3}$ & $(3 / \pi)^{3}$ & $(3 / \pi)^{3}$ \\
\hline$C_{4}$ & $(2 / \pi)^{4}$ & $(2 / \pi)^{5}$ & $(2 / \pi)^{6}$ & $(2 / \pi)^{7}$ \\
\hline$C_{5}$ & $(1 / \pi)^{5}$ & & & $(1 / \pi)^{11}$ \\
\hline$\alpha^{-1}$ value & & $(1 / \pi)^{7}$ & $(1 / \pi)^{9}$ & \\
\hline
\end{tabular}

813 CODATA $\alpha^{-1}$ value: 137.035999084(21).

814 *Each coefficient in the table must be multiplied by a factor of 10 before applying to 815 equation (13).

816 